\title{
Impacto das equipes de saúde bucal da Estratégia da Saúde da Família na saúde bucal de adolescentes do sul do Brasil
}

\author{
Impact of oral health teams of the Family Health Strategy \\ on the oral health of adolescents in the south of Brazil
}

Helenita Corrêa Ely ${ }^{1}$

Claides Abegg ${ }^{2}$

Roger Keller Celeste ${ }^{2}$

Marcos Pascoal Pattussi ${ }^{3}$
${ }^{1}$ Faculdade de Odontologia, Pontifícia Universidade Católica do Rio Grande do Sul. R. Ipiranga 6681/ prédio 6, Partenon. 90619900 Porto Alegre RS Brasil. helenitaely@hotmail.com

${ }^{2}$ Departamento de Odontologia Preventiva e Social, Faculdade de Odontologia, Universidade Federal do Rio Grande do Sul. Porto Alegre RS Brasil. ${ }^{3}$ Programa de PósGraduação em Saúde Coletiva, Universidade do Vale do Rio dos Sinos. São Leopoldo RS Brasil.

\begin{abstract}
The Family Health Strategy (FHS) has produced effective results in health indicators. In this cross-sectional study, the impact of the oral health teams (OHT) of the Family Health Strategy was evaluated on the oral health of 2581 adolescent schoolchildren aged 12 and 15-19 years in 36 municipalities (19 with and 17 without OHT/FHS). Four dentists performed oral examinations. Socioeconomic status, the use of and access to health services were assessed via a structured questionnaire. The presence of the OHT in the FHS were the main independent variables. Outcomes were Decayed, Missing, Filled Teeth (DMFT) and its components, toothache, gingival bleeding, and dental calculus. The data were analyzed by means of negative binomial and Poisson regression. Multilevel analysis was conducted to adjust the outcomes to OHT/FHS and individual variables. In the unadjusted model there was no association between the OHT in the FHS and the outcomes analyzed. After adjustment, young people in areas not covered by the OHT/FHS had almost half of the tooth loss of adolescents from the areas covered ( $R M=0.64$ CI 95\%, 0.43 to 0.94 ). Key words Oral health, Family Health Strategy, Adolescents
\end{abstract}

Resumo A Estratégia da Saúde da Família (ESF) tem demostrado resultados efetivos em indicadores de saúde. Neste estudo transversal foi avaliado o impacto das equipes de saúde bucal (ESB) da ESF na saúde bucal de 2581 adolescentes escolares, com idade de 12 e 15-19 anos de 36 municípios, 19 com e 17 sem ESB/ESF. Quatro dentistas realizaram exames bucais. Situação socioeconômica, uso e acesso aos serviços de saúde foram coletados em questionário estruturado. Presença das ESB na ESF foi a principal variável independente. Os desfechos foram CPOD e seus componentes, dor de dente, sangramento gengival e cálculo dentário. Os dados foram analisados pelas médias das regressões binomiais negativas e Poisson. O efeito da ESB/ESF e de variáveis individuais foi ajustado em modelo multinível. No modelo bruto não houve associação das ESB na ESF com nenhum dos desfechos analisados. Após o ajuste, jovens de áreas não cobertas pela ESB/ESF tiveram quase a metade da perda de dentes dos adolescentes das áreas cobertas ( $R M=0,64$ IC95\%; 0,43-0,94). Palavras-chave Saúde bucal, Estratégia de Saúde da Família, Adolescentes 


\section{Introdução}

A Estratégia da Saúde da Família (ESF) é a principal política do sistema de saúde brasileiro para operar e estruturar a atenção básica nos sistemas locais de saúde, sendo guiada pelos princípios da Atenção Primária à Saúde (APS). Em 2000 ocorre a inclusão das equipes de saúde bucal (ESB) na ESF, como medida reorganizadora das práticas de atenção à saúde bucal no Sistema Único de Saúde, preconizando a adesão aos princípios da atenção primária e aos modelos de vigilância e promoção da saúde ${ }^{1}$. Incentivada com recursos financeiros adicionais ao programa geral, no ano de 2013 um total de 23.092 ESB atendem aproximadamente $39 \%$ da população brasileira em 4.962 municípios². $^{2}$

Estudos desenvolvidos para medir os efeitos da implantação da ESF na saúde da população têm demonstrado sua associação com a redução da mortalidade infantil ${ }^{3}$, declínio de hospitalizações evitáveis ${ }^{4}$, aumento da oferta de trabalho entre os adultos e aumento da matrícula escolar ${ }^{5}$. Em relação à saúde bucal, as análises têm se caracterizado, sobretudo, como pesquisas de avaliação dos processos, isto é, tendo como desfechos indicadores de uso e acesso dos serviços de saúde $\mathrm{d}^{6-9}$. Estudos realizados para avaliar a utilização dos serviços concluíram que áreas cobertas pelas equipes de saúde bucal aumentavam o uso de serviços se comparados aos locais com ausência de serviços, mas não comparadas com áreas cobertas por unidades de saúde tradicionais?. Rocha e Goes ${ }^{8}$ não encontraram associação entre residir ou não em área com ESF e o acesso aos serviços de saúde bucal em Campina Grande, Paraíba, sugerindo dificuldades das populações adscritas ao cuidado regular com a saúde bucal, também observado por Baldani e Antunes9.

Avaliações de resultados com desfechos de saúde bucal são escassas e há poucas evidências de impacto benéfico produzido pelas ESB. Um estudo no Nordeste brasileiro mostrou que o melhor resultado foi obtido para os indicadores de acesso aos serviços e às ações coletivas; enquanto que para morbidade dentária, ações preventivas individuais e cobertura de tratamentos dentários as ESB tenderam a impactos negativos ou a ausência de impacto ${ }^{10}$. Resultados referentes à dor de origem dentária foram igualmente negativos para atuação das $\mathrm{ESB}^{10}$. Outro estudo comparou a prevalência e a intensidade da dor dental entre adolescentes de áreas com e sem Equipe de Saúde Bucal na $\mathrm{ESF}^{11}$. A prevalência de dor dental nos últimos 3 meses foi de $33,7 \%$ nos dois grupos de estudo. No entanto, as chances de um adolescen- te, morador de área onde o tratamento odontológico é oferecido de forma apenas curativa, relatar dor de intensidade média ou alta foi duas vezes maior que entre aqueles que vivem em locais com ações de promoção de saúde inseridas em Unidades da ESF.

Considerando-se o cenário atual, em que avaliações de resultados para desfechos odontológicos são raras, porém necessárias, este estudo se propôs a avaliar o resultado das ESB em indicadores relacionados ao número de dentes cariados, perdidos e restaurados, dor de origem dental, sangramento gengival e cálculo dentário em adolescentes de municípios do Rio Grande do Sul com e sem ESB-ESF.

\section{Método}

Trata-se de um estudo com delineamento transversal. O presente trabalho representa parte de uma pesquisa ${ }^{12}$ que inclui a análise do perfil de saúde bucal de adolescentes escolares de 12 e de 15-19 anos de municípios de pequeno porte do Rio Grande do Sul, cobertos e não cobertos pela ESB na ESF, decorridos oito anos da participação no Levantamento Epidemiológico da saúde bucal na população (SB RS 2002/2003) ${ }^{13}$.

O plano amostral foi realizado em três etapas: amostragem dos municípios, das escolas e dos indivíduos. Outro trabalho anterior realizado por Ely et al. ${ }^{14}$ detalha igualmente a metodologia utilizada neste estudo.

\section{Seleção de Municípios}

Para a seleção dos municípios foi mantida a mesma pré-estratificação em portes utilizada no estudo do SB-RS 2002/2003 ${ }^{13}$ : porte 1 (até 5.000 habitantes); porte 2 (5.001 a 10.000 habitantes); porte 3 (10.001 a 50.000 habitantes); e porte 4 (> 50.000 habitantes). Foram selecionados os municípios segundo os seguintes critérios de inclusão: a) ter participado do SB RS2002/2003; b) nunca teve ESB na ESF ou ter ESB/ESF com tempo de implantação igual ou maior que 5 anos, critério similar ao utilizado por Giovanella et al..$^{15}$; c) cobertura da ESF igual ou maior que $90 \%$ da população. Na sequência procedeu-se a seleção dos municípios com e sem ESB/ESF dentro de cada porte, sendo selecionados 18 municípios no porte 1,12 no porte 2 e 8 no porte 3 , totalizando uma amostra de 38 municípios (no porte 4, todos os municípios apresentavam ausência de ESB/ESF, sendo excluídos 7 municípios). Os critérios para seleção destes municípios foram a macrorregião 
e o porte municipal (Figura 1). Foram enviados ofícios para todos os 38 municípios explicando o projeto e solicitando autorização da Secretaria da Saúde e da Educação dos municípios. Dois municípios do porte 1 foram excluídos após manifestação expressa de recusa na participação da pesquisa. Assim, a amostra final do estudo totalizou 36 municípios de sete macrorregiões do RS e de três portes populacionais, sendo 19 municípios no grupo 1 (com equipes de saúde bucal na ESF) e 17 no grupo 2 (sem equipes de saúde bucal na ESF).

\section{Seleção das escolas}

Inicialmente foram relacionadas todas as 273 escolas de ensino fundamental e médio, urbanas e rurais, públicas e privadas dos 36 municípios da pesquisa segundo dados da Secretaria da Educação do Estado do $\mathrm{RS}^{16}$. Os critérios de inclusão para escolas foram: a) ter ensino fundamental completo; b) ter alunos na faixa etária da pesquisa e; c) localização até $10 \mathrm{~km}$ da sede do município (para escolas rurais e por razões de operacionalidade do projeto). Eram elegíveis 145 escolas, das quais $125(86,62 \%)$ aceitaram participar, sendo 98 urbanas $(78,4 \%)$ e 27 rurais $(21,6 \%)$. Apenas cinco escolas da amostra são de natureza privada, sendo as demais públicas municipais ou estaduais.

\section{Seleção de Indivíduos}

A amostra de indivíduos foi constituída por adolescentes escolares de 12 anos e 15-19 anos,

Municípios Participantes do SB/RS

86 MUNICÍPIOS
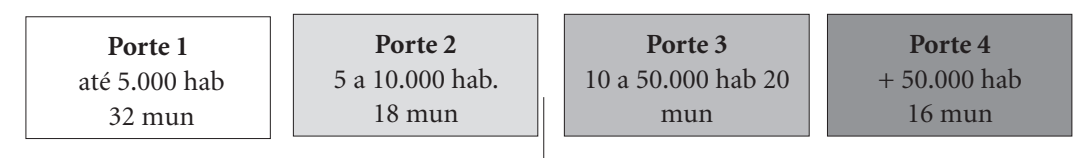

Distribuição 86 municípios por porte populacional e ESF/SB

Distribuição 86 municípios por porte populacional e ESF/SB
\begin{tabular}{|c|c|c|c|c|c|c|c|}
\hline Sem ESB & Com ESB & Sem ESB & Com ESB & Sem ESB & Com ESB & Sem ESB & Com ESB \\
9 & 23 & 6 & 12 & 7 & 13 & 7 & 9 \\
\hline
\end{tabular}
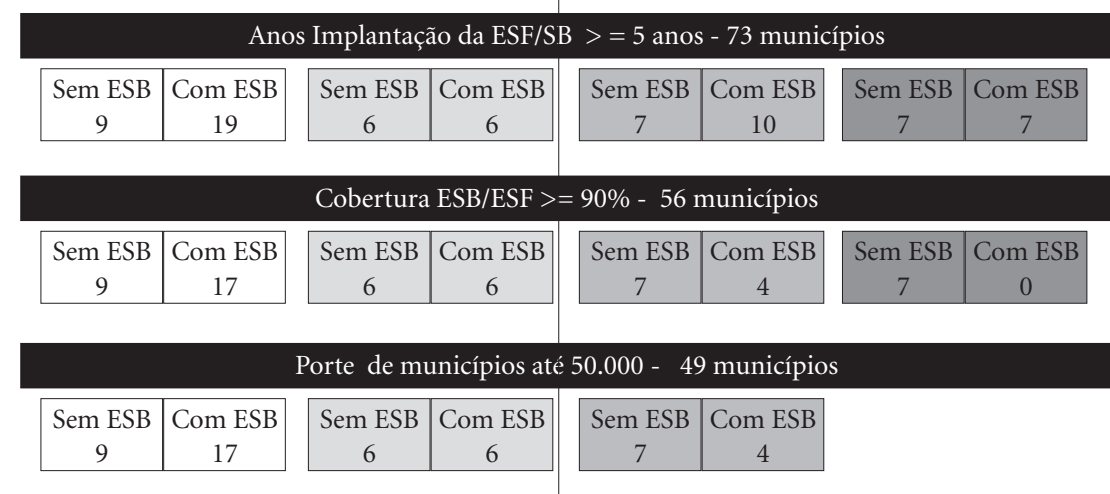

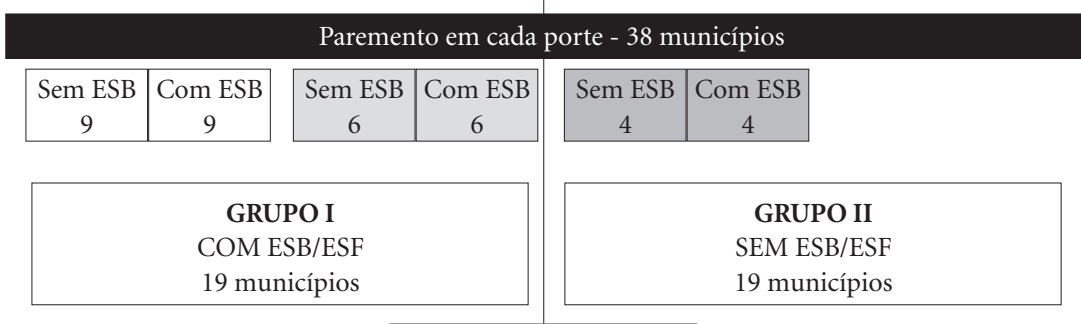

38 municípios participantes do estudo

Figura 1. Processo de seleção dos municípios com aplicação dos critérios de inclusão. 
residentes na área urbana ou rural dos municípios da pesquisa. Para o cálculo amostral foram utilizadas estimativas das médias CPOD de adolescentes de 15 a 19 anos do SB RS 2003 para ambos os grupos, pois o uso das médias CPOD de 12 anos representavam uma amostra menor. Assim, para as cidades cobertas pelas ESB da ESF, foi usada a média 7,67 e desvio padrão de 2,33 e para cidades não cobertas pelas ESB/ESF, a média 7,16 e desvio padrão 1,97, com um nível significância de 0,05 e um poder de $95 \%$. O tamanho amostral calculado foi um total de 465 indivíduos em cada grupo. Considerando-se ainda uma taxa de não resposta de $20 \%$ e um efeito de delineamento de 1,5, a amostra mínima necessária foi de 790 indivíduos em cada grupo etário e em cada situação, representando uma amostra final de 3160 adolescentes. Esta amostra foi distribuída de forma proporcional ao tamanho da população das idades estudadas e ao número de escolas de cada município amostral. Considerando a baixa densidade populacional de alguns municípios (porte 1), definiu-se como regra de decisão deste estudo, a inclusão de todos os alunos da faixa etária em análise, quando o total de alunos da escola destas idades (12 e 15-19 anos) fossem até duas vezes o número total da amostra naquela escola. Nas demais situações procedeu-se a uma seleção casual sistemática para amostragem dos indivíduos nas escolas, a partir de listas de todos alunos da idade fornecidas pelas escolas.

\section{Coleta dos dados}

Os exames foram realizados por quatro cirurgiões dentistas auxiliados por anotadores devidamente treinados. O processo de calibração interexaminadores ocorreu previamente à coleta de dados com simulação de situações a serem identificadas na pesquisa e discussão do registro até o consenso. Após, foi operacionalizado um estudo piloto em um município para avaliação e adequação dos instrumentos. Os exames clínicos foram realizados nas escolas, no período entre julho e dezembro de 2011. Os índices e critérios para os agravos analisados seguiram recomendação do Ministério da Saúde ${ }^{17}$. Durante a pesquisa foi avaliada a reprodutibilidade dos exames clínicos intraexaminadores pela duplicação dos mesmos em 5\% da amostra, cuja consistência foi analisada por meio do coeficiente Kappa ponderado (k $=0,91-0,94)$ para condição da coroa. A confiabilidade dos exames entre examinadores, avaliada na calibração antes da coleta de dados em campo apresentou valores kappa $\geq 0,76(0,76-0,94)$ para todas as condições avaliadas.
Os dados sobre situação socioeconômica, autopercepção, acesso e uso dos serviços foram coletados através de um questionário semiestruturado enviado ao domicílio para preenchimento pelos pais ou responsáveis dos escolares de 12 anos. Os adolescentes de 15 a 19 anos, após explicação da pesquisa em sala de aula, respondiam individualmente antes dos exames clínicos. Os formulários foram reenviados aos pais até duas vezes e, na permanência da não devolução, o aluno selecionado foi excluído da amostra e computado na taxa de não reposta do estudo.

\section{Desfechos}

Foram utilizados desfechos em nível individual, modelados como variáveis de contagens, derivados do índice CPO. Os desfechos relativos à saúde bucal foram selecionados por serem passíveis de impacto na mudança a partir da implantação das ESB/ESF: a) número de dentes cariados, b) número de dentes restaurados; c) número de dentes perdidos; d) índice CPO (número de cariados, perdidos e obturados). Sangramento gengival e presença de cálculo dentário foram registrados segundo constatação de presença ou ausência no sextante e sumarizados por indivíduos segundo prevalência de pelo menos um sextante com o agravo. Prevalência de dor dental foi sumarizada a partir das respostas ao questionamento: "Você sentiu dor de dente nos últimos seis meses?"

\section{Variáveis Independentes}

A principal variável explicativa do estudo foi a presença das equipes de saúde bucal na estratégia da saúde da família implantada nos municípios. A pesquisa desenvolveu-se em um subconjunto de municípios com um mínimo de $90 \%$ de cobertura das equipes de saúde bucal implantadas na ESF há pelo menos 5 anos antes da coleta de dados (Grupo 1), e em outro (Grupo 2) sem nenhuma equipe de saúde bucal implantada nas ESF. O grupo 2 foi considerado como um modelo tradicional de atenção à saúde bucal.

As variáveis independentes incluídas no modelo foram apenas aquelas consideradas como potenciais fatores de confusão. Foram incluídas no nivel de indivíduos: sexo (masculino/feminino), idade (12, 15 a 19 anos), renda domiciliar equivalente ${ }^{18}$, escolaridade da mãe medida em anos de estudo ( $0-4$ anos para ensino fundamental incompleto; 5 a 8 , ensino fundamental; 9 a 11 ,ensino médio e 12 ou mais para ensino), o tempo da última visita ao dentista (nunca foi, 3 
anos ou mais, um ou 2 anos, menos de um ano, não sabe) o motivo da visita (nunca foi, não sabe, revisão/rotina, tratamento) e o local da última consulta (nunca foi, serviço público/SUS, plano privado/particular). Foram incluídas no nível municipal: o PIB per capita municipal (em Reais), anos de fluoretação da água de abastecimento público, porte populacional (até 5 mil habitantes, de 5 a 10 mil habitantes, de 10 a 50mil habitantes) e percentual de população rural do município, cujas fontes estão descritas em Ely et al. ${ }^{14}$.

\section{Análise Estatística}

Sobredispersão foi avaliada utilizando-se a estatística de deviance, conforme Hardin e Hil$\mathrm{be}^{19}$. Análises preliminares mostraram evidências de sobredispersão para três desfechos: número de dentes cariados (sobredispersão $=2.1$ ), número de dentes obturados (sobredispersão $=2,8$ ) e o índice CPOD (sobredispersão = 3,2). Desfechos com sobredispersão foram modelados utilizando regressão binomial negativa, e o número de dentes perdidos foi modelado com regressão de Poisson, usando como estimador Penalised Quasi-Likelihood de segunda ordem (PQL2). Para os desfechos de dentes obturados e cariados, o logaritmo natural dos dentes presentes foi utilizado como offset.

Para avaliar o efeito do município ter ESB/ ESF, foram utilizados modelos multiníveis com intercepto aleatório. Indivíduos foram alocados como unidades de primeiro nível, agrupados nos seus respectivos municípios, que são as unidades de segundo nível. A variância de segundo nível foi calculada segundo Método D proposto por Goldstein et al. ${ }^{20}$, em que a variância de primeiro nível é fixa no valor de 3.29 (๗).

Foram realizadas análises para avaliar a interação entre o local de consulta (SUS versus plano/privado) e o efeito do município ter ESB/ESF. Para todos os desfechos, nas análises brutas, contendo 3 coeficientes ( 2 efeitos principais de ter ido no SUS e morar num município com ESB/ ESF, e um termo de interação), a direção da associação foi igual dentre usuário e não usuário do SUS, bem como nenhum termo de interação foi significativo $(p>0.05)$. Assim, todas as análises foram realizadas com a amostra inteira.

As análises para modelos multiníveis com intercepto aleatório foram realizadas no software MlwiN 2.27, as demais foram feitas no software Stata 11.2.

\section{Aspectos Éticos}

O projeto foi submetido e aprovado pelo Comitê de Ética em Pesquisa da Universidade Federal do Rio Grande do Sul e do Comitê de Ética em Pesquisa da Escola de Saúde Pública da Secretaria de Estado da Saúde do Rio Grande do Sul e apoiado com recursos da Fundação de Amparo a Pesquisa do Rio Grande do Sul. Indivíduos, municípios e escolas participantes do estudo receberam informações sobre a pesquisa e assinaram o termo de consentimento prévio à coleta de dados. Para os escolares de 12 anos, o termo foi assinado pelos pais ou responsáveis após serem esclarecidos. Após o exame epidemiológico, cada participante foi informado sobre sua saúde bucal e, na presença de necessidade de tratamento, referenciado para o serviço municipal de odontologia. Um relatório final sobre a situação dos municípios e macrorregiões foi enviado para a Secretaria da Saúde do Estado e disponibilizado para consulta pública.

\section{Resultados}

Participaram do estudo 3531 indivíduos da faixa etária de 12 e de 15 a 19 anos, de 19 municípios com equipes de saúde bucal na ESF iniciada entre 2002 e 2004, e outros 17 municípios sem ESB/ ESF com modelos tradicionais de atenção à saúde bucal. Desta amostra, 2581 adolescentes foram efetivamente analisados, correspondendo a uma taxa de resposta de $73 \%$. Os motivos para esta redução foram perdas por ausência de resposta em alguns itens do formulário socioeconômico, especialmente em relação à renda, absenteísmo à escola no dia da pesquisa e não autorização para participação na pesquisa. A variável renda domiciliar apresentou o maior número de valores missing $(\mathrm{n}=687)$.

A Tabela 1 apresenta a distribuição da amostra nas duas situações de pesquisa na qual se verifica uma distribuição homogênea entre os dois grupos de análise, em relação aos grupos etários, sexo e escolaridade da mãe, cuja média de anos de estudo foi de 13 anos ( $\mathrm{dp} \pm 23,9)$ para os dois grupos. A renda média declarada das famílias situou-se na faixa entre $\mathrm{R} \$ 500,00$ a $\mathrm{R} \$ 1.500,00$, com um valor médio de renda per capita equivalente de $\mathrm{R} \$ 804,84$ ( $\mathrm{dp} \pm 829,27)$, valor acima do salário mínimo do Estado em 2011 (R\$ 610,00). A maioria dos adolescentes foi ao dentista no último ano $(67 \%)$ em Unidade de Saúde do SUS (52\%) para revisão de rotina (52\%) e moram em cidades abastecidas com águas fluoretadas 
Tabela 1. Distribuição da amostra de adolescentes escolares, em valores absolutos e proporcionais em 36 municípios segundo a presença ou ausência de equipes de saúde bucal na Estratégia de Saúde da Família. RS, 2011.

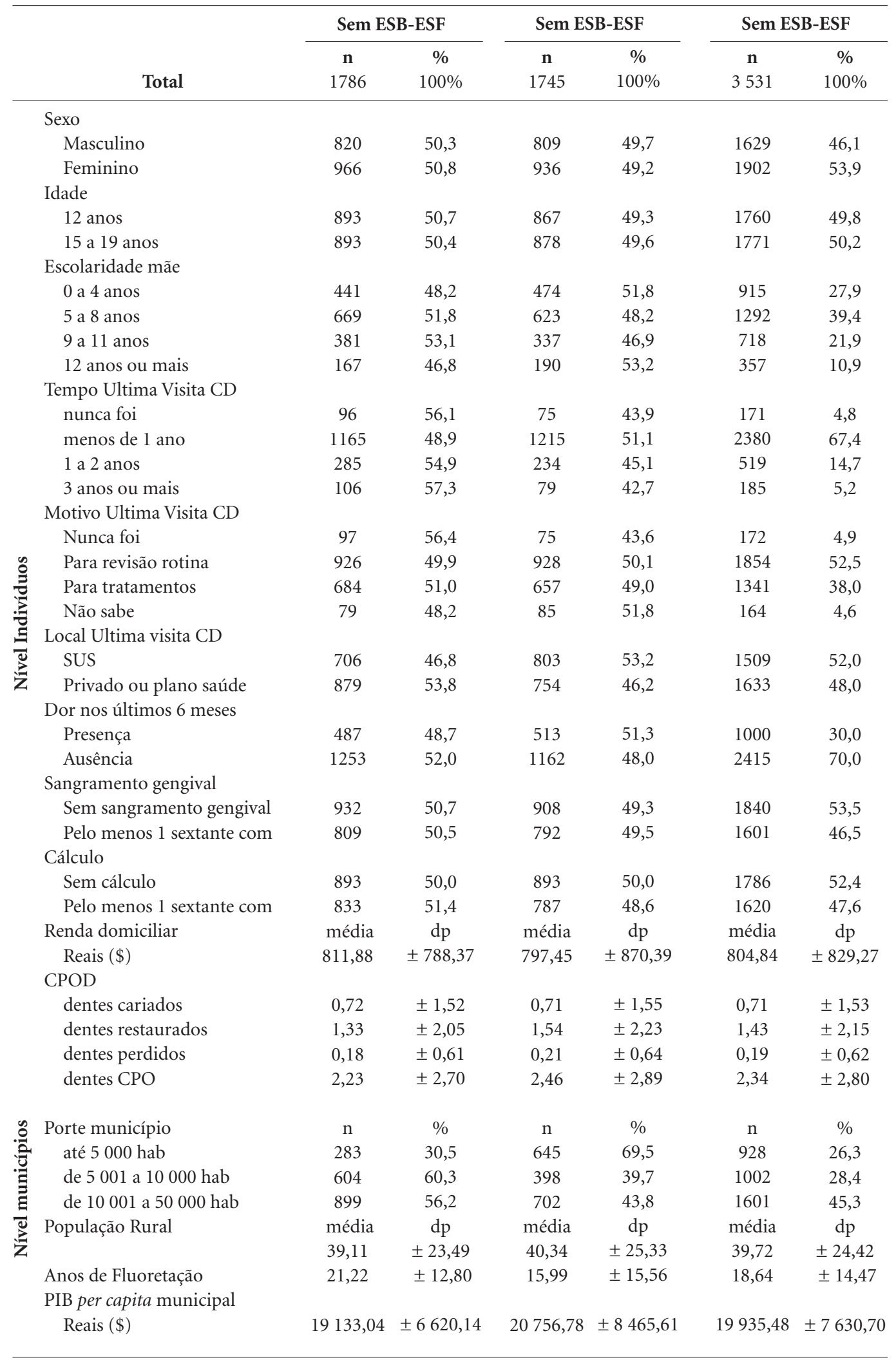


(82\%). O número médio de dentes cariados, restaurados, perdidos e médias CPOD está descrito na Tabela 1, para as duas situações de pesquisa.

A Tabela 2 apresenta os valores brutos, ajustados e intervalos de confiança ao nível de 95\% das razões de média para cobertura das equipes de saúde bucal na ESF em modelos de regressão binomial negativa. Isso significa que os resultados obtidos podem ser interpretados sem interferência de possíveis efeitos de confundimento. No modelo bruto não se observou associação das ESB com nenhum dos desfechos analisados. No modelo ajustado, mantendo constantes as variáveis individuais e municipais, "dentes perdidos" teve um efeito significativo para os adolescentes de municípios sem ESB/ESF. Após o ajuste, jovens de áreas não cobertas pela ESB/ESF tiveram quase a metade da perda de dentes dos adolescentes das áreas cobertas ( $\mathrm{RM}=0,64 \mathrm{IC} 95 \%$; 0,43-0,94). Nos demais desfechos analisados, dentes cariados, dentes restaurados, médias CPO, não se observou diferenças significativas entre os adolescentes das duas situações analisadas.
$\mathrm{Na}$ análise dos desfechos com as variáveis individuais, dentes cariados associaram-se significativamente com sexo feminino, escolaridade materna, local e tempo da última consulta ao dentista. Ser do sexo feminino (RM 0,74; IC95\% 0,63-0,87) e ter mãe com ensino médio (RM 0,49; IC 95\% 0,38-0,63) ou superior (RM 0,36; IC 95\% 0,25-0,51) associa-se com ter menor número de dentes cariados. Por outro lado, ter feito a última consulta ao dentista no SUS e há mais de três anos, associou-se com mais cárie não tratada (dados não apresentados). Observou-se também que ter idade maior e ter consultado o dentista no último ano está associado com maior número de dentes restaurados. Ter realizada ultima consulta no SUS (RM 0,75; IC 95\% 0,67-0,84) e há mais tempo (+3anos RM 0,55; IC 0,43-0,71), associa-se significativamente com menor número de dentes restaurados. Ter mais idade também representa maior perda dentária e maior média CPOD. Entretanto, ter mãe com escolaridade de nível médio (RM 0,64; IC 0,45-0,91) ou nível superior (RM 0,17; IC 0,08-0,36) e ter feito

Tabela 2. Razão de médias (RM) e razão de chances (OR) de cobertura das Equipes de Saúde Bucal da Estratégia da Saúde da Família para diferentes desfechos, em modelo de regressão multinível para 2581 indivíduos de 36 municípios do Rio Grande do Sul. 2011.

\begin{tabular}{|c|c|c|c|c|}
\hline & \multicolumn{2}{|c|}{ Modelo Bruto } & \multicolumn{2}{|c|}{ Modelo Ajustado* } \\
\hline & RM & IC95\% (LI -LS) & RM & IC95\% (LI-LS) \\
\hline \multicolumn{5}{|l|}{ No Dentes Cariados } \\
\hline Presença de ESB & 1 & & 1 & \\
\hline Ausência de ESB & 0,89 & $(0,64-1,22)$ & 1,07 & $(0.88-1,29)$ \\
\hline \multicolumn{5}{|c|}{ No Dentes Restaurados } \\
\hline Presença de ESB & 1 & & 1 & \\
\hline Ausência de ESB & 0,93 & $(0,67-1,28)$ & 0.99 & $(0,75-1,29)$ \\
\hline \multicolumn{5}{|l|}{ No Dentes Perdidos } \\
\hline Presença de ESB & 1 & & 1 & \\
\hline Ausência de ESB & 0,72 & $(0,44-1,17)$ & 0,64 & $(0,43-0,94)$ \\
\hline \multicolumn{5}{|l|}{ CPOD } \\
\hline Presença de ESB & 1 & & 1 & \\
\hline \multirow[t]{2}{*}{ Ausência de ESB } & 0,89 & $(0,71-1,10)$ & 0,97 & $(0,79-1,18)$ \\
\hline & OR & IC95\% & OR & IC95\% \\
\hline \multicolumn{5}{|c|}{ Presença de dor nos últimos 6 meses } \\
\hline Presença de ESB & 1 & & 1 & \\
\hline Ausência de ESB & 0,90 & $(0.72-1.13)$ & 0,87 & $(0.67-1.12)$ \\
\hline \multicolumn{5}{|c|}{ Ao menos um sextante com sangramento } \\
\hline Presença de ESB & 1 & & 1 & \\
\hline Ausência de ESB & 1,23 & $(0.80-1.88)$ & 1,17 & $(0.73-1.88)$ \\
\hline \multicolumn{5}{|c|}{ Ao menos um sextante com cálculo dental } \\
\hline Presença de ESB & 1 & & 1 & \\
\hline Ausência de ESB & 1,15 & $(0.77-1.71)$ & 1,07 & $(0.70-1.63)$ \\
\hline
\end{tabular}

${ }^{*}$ Nível individual: Sexo, idade, renda domiciliar equivalente, escolaridade da mãe, tempo da última consulta ao dentista, motivo e local da consulta ao dentista. ${ }^{*}$ Nível municipal: PIB per capita, anos de fluoretação, porte populacional, \% população rural. 
sua ultima visita ao dentista há mais de três anos (RM 0,34; IC 0,18-0,67) está associado com menos dentes perdidos, bem como menor média CPOD. Para o desfecho dor de dente nos últimos seis meses não foram observadas diferenças significativas entre os dois grupos, tanto no modelo bruto como no ajustado (Tabela 2). A prevalência de pelo menos um sextante com sangramento (RM 1,17; IC 0,73-1,88) e a presença de um sextante com cálculo (RM 1,15; IC 0,7-1,63) é maior no grupo sem ESB, mas as diferenças não foram estatisticamente significativas.

Os resultados acerca da variância de segundo nível mostram que a variabilidade atribuível à diferenças entre os municípios (Tabela 3) é maior para o desfecho de dentes perdidos, mesmo após controle por variáveis individuais $(11.9 \%, \mathrm{p}<$ 0.01 ), enquanto que para dor de dente as diferenças entre municípios não são significativas.

\section{Discussão}

A análise de impacto da implantação das ESB/ESF em municípios com menos de 50mil habitantes do RS na saúde bucal de adolescentes observou diferenças significativas, comparando municípios com e sem equipes de saúde bucal no modelo da ESF. Dos desfechos avaliados, apenas um foi estatisticamente significativo: adolescentes de municípios sem ESB/ESF têm uma média de dentes perdidos 36\% menor (RM = 0,64, IC95\%: 0,43$0,94)$ do que jovens de municípios cujo modelo de atenção incluiu saúde bucal na ESF.

Considerando as diretrizes da Política Nacional de Atenção Básica ${ }^{21}$ e a proposta de reorientação do processo de trabalho em saúde bucal para a Estratégia de Saúde da Família, seria esperado que os municípios com ESB/ESF, ou ao menos os usuários desses serviços, apresentassem desfechos melhores, tais como, maior número de dentes restaurados, menos dor dentária, menor perda dentária. Evidências mostram que indivíduos de municípios gaúchos que produzem menos atividades preventivas possuem 1,76 vezes mais chances de ter cáries, embora o efeito não tenha sido observado para a experiência total de cáries $^{22}$. Os resultados obtidos neste estudo são semelhantes àqueles descritos por Pereira et al. ${ }^{10}$, em que indivíduos cobertos e não cobertos com ESB não apresentaram diferenças em desfechos de saúde bucal, em 12 cidades da região Nordeste. Por outro lado, estudos com desfechos que não incluem saúde bucal, têm demonstrado que o modelo preconizado pela ESF é uma estratégia efetiva para promover saúde em áreas mais vulneráveis ${ }^{23,24}$.

É possível que condições específicas dos municípios investigados possam explicar em parte os resultados encontrados nesta pesquisa. Para que as ESB tenham algum efeito na saúde da população é necessário que a mesma utilize os serviços. No presente estudo, a população coberta por ESB/ESF apresentou maior uso dos serviços, porém, aproximadamente $25 \%$ dos jovens usaram os serviços há mais de 1 ano, ou nunca usaram, e dentre os usuários, 52\% referiu não ter usado o SUS na ultima visita ao dentista. Tal achado está em consonância com um estudo que mostra que, nas capitais brasileiras, o uso de serviços odontológicos tem declinado entre os jovens sendo as melhorias em saúde bucal uma das possíveis razões $^{25}$. A literatura relata que a ampliação do número de equipes de saúde bucal no Brasil tem contribuído para o aumento do uso e do acesso aos serviços odontológicos ${ }^{6,9,26}$ e maior satisfação

Tabela 3. Coeficiente de Partição de Variância atribuível ao nível contextual e significância estatística da variância de segundo nível em 3 modelos para diferentes desfechos em regressão multinível de 36 municípios do Rio Grande do Sul. 2011.

\begin{tabular}{|c|c|c|c|c|c|c|}
\hline & \multicolumn{2}{|c|}{ Modelo Bruto } & \multicolumn{2}{|c|}{$\begin{array}{c}\text { Mod. Ajust. } \\
\text { (vars individuais) }\end{array}$} & \multicolumn{2}{|c|}{$\begin{array}{l}\text { Mod. Ajust. } \\
\text { (todas vars) }\end{array}$} \\
\hline & VPC (\%) & p-valor & VPC (\%) & p-valor & VPC (\%) & p-valor ${ }^{*}$ \\
\hline No Dentes Cariados & 6.6 & $<0.01$ & 1.9 & 0.04 & 1.1 & 0.18 \\
\hline No Dentes Restaurados & 6.2 & $<0.01$ & 5.6 & $<0.01$ & 3.7 & $<0.01$ \\
\hline No Dentes Perdidos & 12.5 & $<0.01$ & 11.9 & $<0.01$ & 7.2 & $<0.01$ \\
\hline CPOD & 3.2 & $<0.01$ & 2.7 & $<0.01$ & 1.9 & $<0.01$ \\
\hline Presença de dor no últimos 6 meses & 1.8 & 0.07 & 1.6 & 0.14 & 1.1 & 0.29 \\
\hline Ao menos um sextante com sangramento & 10.0 & $<0.01$ & 11.5 & $<0.01$ & 11.1 & $<0.01$ \\
\hline Ao menos um sextante com cálculo dental & 8.8 & $<0.01$ & 9.7 & $<0.01$ & 8.2 & $<0.01$ \\
\hline
\end{tabular}

* Significância estatística da variância de segundo nível segundo teste de Wald. 
dos usuários ${ }^{27}$. É necessário que se avalie o quanto seria necessário aumentar a cobertura e o uso para impactar na saúde da população.

Outra possível explicação para a ausência de efeito é que os processos de trabalho das equipes de saúde bucal dos municípios com e sem ESB/ ESF sejam similares. Um estudo em municípios do Estado de Pernambuco relata que os serviços odontológicos existentes (do modelo tradicional) foram transformados em equipes de saúde bucal $^{28}$. Se essa lógica foi adotada também nos municípios desta amostra, a expansão do acesso pode ter resultado em um aumento de procedimentos mutiladores, explicando porque a presença de ESB/ESF esteve associada apenas com perda dental.

A implantação da ESF nos municípios oferece aos gestores múltiplas possibilidades de captação de recursos financeiros federais para expansão da atenção básica. No entanto, muitos municípios de pequeno porte têm dificuldade de organizar programas de educação permanente para os trabalhadores e estruturar a rede local ou regional para a continuidade dos tratamentos aos níveis de maior complexidade ${ }^{29}$, condições necessárias para superar um modelo tradicional de atenção às demandas reprimidas e grupos vulneráveis. Como consequência, permanece o desafio de ressignificar as práticas, humanizar o cuidado no ato vivo do trabalho em saúde ${ }^{30}$ e dar resolutividade aos problemas de saúde trazidos pelas comunidades adscritas.

Há necessidade de se repensar a maneira como os recursos humanos para a saúde são formados ${ }^{31}$. Embora os atributos desejáveis aos trabalhadores de saúde estejam descritos nas Diretrizes Curriculares Nacionais para os cursos da área da saúde, observa-se ainda um distanciamento entre os princípios norteadores do Sistema Único de Saúde e o sistema de ensino superior ${ }^{32}$. A abordagem social, a humanização do cuidado

\section{Colaboradores}

HC Ely e C Abegg participaram da concepção do estudo, revisão de literatura, coleta dos dados, interpretação dos resultados e redação do manuscrito; RK Celeste e MP Pattussi contribuíram com a análise dos dados, interpretação dos resultados e revisão final do manuscrito. e o uso de tecnologia apropriada, orientado por evidências científicas têm sido pouco discutidos na formação dos profissionais de saúde ${ }^{33}$.

Uma das limitações do presente estudo refere-se ao fato do mesmo não ter avaliado a influência de variáveis decorrentes de comportamentos individuais, hábitos e estilos de vida. Por outro lado, tem sido argumentado que estudos em saúde pública devem ir para além dos aspectos individuais com foco tanto em indivíduos como em lugares, pois muito dos comportamentos individuais são influenciados ou moldados pelo ambiente onde as pessoas vivem.

Neste sentido, o presente estudo contribui com a literatura sobre o tema ao adotar um delineamento e análise dos dados adequados, levando em consideração tanto a inclusão de variáveis contextuais, relativas aos municípios, como individuais e fatores de confundimento. Novos estudos enfocando aspectos como a natureza e a dinâmica do trabalho das equipes de saúde bucal e seu perfil, em municípios com ESB e sem ESB, poderiam melhor clarificar os resultados aqui apresentados.

Este estudo sugere que a expansão do acesso com a inserção de equipes de saúde bucal, não se traduziu em melhor saúde bucal. Por outro lado, confirma a importância da determinação social na saúde bucal de adolescentes, como tem sido demonstrado em estudos anteriores. Esses resultados podem estimular a revisão dos processos de trabalho exercidos nas ESB, a revisão das intervenções na linha de cuidado e do planejamento em saúde e ações que efetivamente alterem as condições de saúde e a melhoria da qualidade de vida da população. Considerando o contexto atual, com grande alocação de recursos nos estados e municípios, é crescente a necessidade de práticas de saúde baseadas em evidência, de investigações que permitam melhor quantificar e qualificar o impacto das intervenções e das políticas em saúde pública adotadas no Brasil.

\section{Referências}

1. Brasil. Ministério da Saúde (MS). Portaria N. ${ }^{\circ}$ 1.444, de 28 de dezembro de 2000. Estabelece incentivo financeiro para a reorganização da atenção à saúde bucal prestada nos municípios por meio do Programa de Saúde da Família. Diário Oficial da União 2000; 29 dez.

2. Brasil. Ministério da Saúde (MS). Histórico de Cobertura da Saúde da Família. 2013 [acessado 2014 jan 14]. Disponível em: http://dab.saude.gov.br/portaldab/historico _cobertura_sf.php 
3. Aquino R, de Oliveira NF, Barreto ML. Impact of the family health program on infant mortality in Brazilian municipalities. Am J Public Health 2009; 99(1):87-93.

4. Macinko J, Dourado I, Aquino R, Bonolo Pde F, LimaCosta MF, Medina MG, Mota E, de Oliveira VB, Turci MA. Major expansion of primary care in Brazil linked to decline in unnecessary hospitalization. Health Aff (Millwood) 2010; 29(12):2149-2160.

5. Rocha R, Soares RR. Evaluating the impact of community-based health interventions: evidence from Brazil's Family Health Program. Health Econ 2010; 19(Supl.): 126-158.

6. Soares FF, Figueiredo CR, Borges NC, Jordao RA, Freire MC. Oral health teamwork in Brazilian family healthcare strategy: an analysis of studies published between 2001 and 2008. Cien Saude Colet 2011; 16(7):3169-3180.

7. Pereira CR, Patricio AA, Araujo FA, Lucena EE, de Lima $\mathrm{KC}$, Roncalli AG. Inclusion of oral health teams in the Family Health Program and its impact on the use of dental services. Cad Saude Publica 2009; 25(5):985-996.

8. Rocha RACP, Goes PS. Comparison of access to Oral Health Services between areas covered and not covered by the Family Health Program in Campina Grande, Paraiba State, Brazil. Cad Saude Publica 2008; 24(12):2871-2880.

9. Baldani MH, Antunes JL. Inequalities in access and utilization of dental services: a cross-sectional study in an area covered by the Family Health Strategy. Cad Saude Publica 2011; 27(Supl. 2):S272-S283.

10. Pereira CR, Roncalli AG, Cangussu MC, Noro LR, Patricio AA, Lima KC. Impact of the Family Health Strategy: an analysis in cities in Northeast Brazil with more than 100,000 inhabitants. Cad Saude Publica 2012; 28(3):449-462.

11. Carvalho JC, Rebelo MA, Vettore MV. Dental pain in the previous 3 months in adolescents and Family Health Strategy: the comparison between two areas with different to oralhealth care approaches. Cien Saude Colet 2011; 16(10):4107-4114.

12. Ely HC. Determinantes sociais e saúde bucal de adolescentes de municípios com e sem estratégia da saúde da família [tese]. Porto Alegre: Universidade Federal do Rio Grande do Sul; 2014.

13. Rio Grande do Sul. Secretaria Estadual da Saúde (SES). Condições de saúde bucal da população brasileira: Ampliação da Amostra para o Rio Grande do Sul - Relatório Final. Porto Alegre: SES; 2003.

14. Ely HC, Abegg C, Rosa AR, Pattussi MP. Redução da cárie dentária em adolescentes: distribuição temporal e espacial em 36 municípios do Sul do Brasil 2003 e 2011. Epidemiol. Serv. Saúde 2014; 23(3):421-434.

15. Giovanella L, de Mendonca MH, de Almeida PF, Escorel S, Senna MC, Fausto MC, Delgado MM, de Andrade CL, da Cunha MS, Martins MI, Teixeira CP. Family health: limits and possibilities for an integral primary care approach to health care in Brazil. Cien Saude Colet 2009; 14(3):783-794.

16. Rio Grande do Sul, Secretaria da Educação. Censo Escolar 2009 - Dados Finais. 2010 [acessado 2011 jan 31]. Disponível em: http://www.educacao.rs.gov.br/pse/html /estatisticas.jsp?ACAO = acao 1

17. Brasil. Ministério da Saúde. Secretaria de Atenção à Saúde. Coordenação Nacional de Saúde Bucal. Projeto SB Brasil 2010. Pesquisa Nacional de Saúde Bucal: manual da equipe de campo [Internet]. [acessado 2012 out 17]. Disponível em: http://dab.saude.gov.br/cnsb/ sbbrasil/ arquivos/SBBrasil2010_Manual_Equipe_Campo.pdf
18. Celeste RK, Bastos JL. Mid-point for open-ended income category and the effect of equivalence scales on the income-health relationship. Rev Saude Publica 2013; 47(Supl. 3):168-174.

19. Hardin J, Hilbe J. Generalized Linear Models and Extensions. College Station: Stata Press; 2001.

20. Goldstein H, Browne W, Rasbash J. Partitioning Variation in Multilevel Models. Understanding Statistics 2002; 1(4):223-231.

21. Brasil. Ministério da Saúde (MS). Departamento de Atenção Básica. Secretaria de Atenção à Saúde. Coordenação Nacional de Saúde Bucal. Diretrizes da Politica Nacional de Saúde Bucal. Brasília: MS; 2004.

22. Celeste RK, Nadanovsky P, Leon AP. Association Between Public Dental Service Preventive Procedures and Caries Prevalence. Rev Saude Publica 2007; 41(5):830-838.

23. Kusma SZ, Moyses ST, Moyses SJ. Health promotion: perspectives for evaluation of oral health in primary healthcare. Cad Saude Publica 2012; 28(Supl.):s9-s19.

24. Organização Panamericana de la Salud (OPAS). La renovación de atención primaria de salud en las Américas. Documento de posición de la Organización Panamericana de la Salud/Organización Mundial de la Salud. Washington: OPAS; 2007.

25. Celeste RK, Nadanovsky P, Fritzell J. Trends in socioeconomic disparities in the utilization of dental care in Brazil and Sweden. Scand J Public Health 2011; 39(6):640-648.

26. Celeste RK, Vital JF, Junger WL, Reichenheim ME. Time series analysis of dental care procedures in Brazilian public services, 1994-2007. Cien Saude Colet 2011; 16(11):4523-4532.

27. Anjos FS, Mestriner SF, Bulgarelli AF, Pinto IC, Mestriner Junior W. Equipes de saúde bucal no Brasil: avanços e desafios. Cienc Cuid Saude 2011; 10(3):601-607.

28. Lourenco EC, Silva AC, Meneghin Mde C, Pereira AC. The insertion of oral health services in the Family Health Program at Minas Gerais State, Brazil. Cien Saude Colet 2009; 14(Supl. 1):1367-1377.

29. da Silva SF, de Lima Martelli PJ, Sa DA, de Cabral AP, Pimentel FC, da Silva Monteiro I, Macedo CL. Analysis of the progress of the oral health teams inserted in the Family Health Strategy in Pernambuco, Northeast Region, Brazil, 2002 until 2005. Cien Saude Colet 2011; 16(1):211-220.

30. Merhy EE. O SUS e um dos seus dilemas: mudar a gestão e a lógica do processo de trabalho em saúde (um ensaio sobre a micropolítica do trabalho vivo). In: Teixeira SF, editor. Democracia e Saúde. São Paulo: Cebes/ Lemos; 1998. p. 125-141.

31. Matos PE, Tomita NE. Oral health in the Brazilian Family Health Program: from the university to training centers. Cad Saude Publica 2004; 20(6):1538-1544.

32. Almeida-Filho N. Higher education and health care in Brazil. Lancet 2011; 377(9781):1898-900.

33. Pezzato LM, L'Abbate S, Botazzo C. The production of micro-policies in the work process in oral health: a socio-analytical approach. Cien Saude Colet 2013; 18(7):2095-2104.

Artigo apresentado em 08/05/2015

Aprovado em 22/10/2015

Versão final apresentado em 24/10/2015 